\title{
On Comparing Adams and Natural Spline Multistep Formulas
}

\author{
By David R. Hill*
}

\begin{abstract}
This paper presents two techniques for the comparison of Adams formulas and methods based on natural splines. A rigorous foundation to the claim that a pth order natural spline formula produces better results than a $p$ th order Adams method, but not quite as good as a $(p+1)$ st order Adams formula is given for $p=2,3,4$, which suggests the general case.
\end{abstract}

1. Introduction. A comparison of the numerical solution of ordinary initial value problems using Adams type formulas and formulas based on natural splines was presented in [1], while a similar comparison for delay differential equations was done in [4]. In both instances, the numerical evidence for a class of prototype problems prompted a statement of the following type: A $p$ th order natural spline scheme was superior to a $p$ th order Adams formula, but not quite as good as the $(p+1)$ st order Adams method. The purpose of this note is to present two techniques that show the conclusion is valid without relying upon numerical observations. One such technique was given in [1] which compared the $L-2$ norms of the Peano kernels for each class of methods.

2. Principal Truncation Error. We shall use the notation of Henrici [3] and consider the solution of an ordinary initial-value problem

$$
y^{\prime}=f(x, y), \quad y(a)=\eta
$$

using linear multistep formulas of the form

$$
\sum_{i=0}^{k} \alpha_{i} y_{n+i}=h \sum_{i=0}^{k} \beta_{i} f_{n+i}, \quad n=0,1,2, \ldots,
$$

where $k$ is a fixed integer, $f_{m} \equiv f\left(x_{m}, y_{m}\right)$ and $y_{m} \approx y\left(x_{m}\right)$, the true solution of (1).

A first crude measure of accuracy of formulas given by (2) is the order of the method; see [3, p. 221]. We shall say that a linear multistep formula (2) is of order $p$ if $C_{0}=C_{1}=\cdots=C_{p}=0$, but $C_{p+1} \neq 0$, where

$$
\begin{aligned}
& C_{0}=\sum_{i=0}^{k} \alpha_{i}, \quad C_{1}=\sum_{i=0}^{k}\left(i \alpha_{i}-\beta_{i}\right), \ldots, \\
& C_{q}=\frac{1}{q !} \sum_{i=1}^{k} i^{q} \alpha_{i}-\frac{1}{(q-1) !} \sum_{i=1}^{k} i^{q-1} \beta_{i}, \quad q=2,3, \ldots
\end{aligned}
$$

Received February 20, 1974.

AMS (MOS) subject classifications (1970). Primary $65 \mathrm{~L} 05$.

*This paper is based on portions of the author's doctoral thesis written under the supervision of Professor George D. Byrne at the University of Pittsburgh. 
We shall call $C_{p+1}$ the principal truncation error coefficient for a method of order $p$. As noted in [3], (see also [5]) the principal truncation error coefficient should not be used as a finer measure of accuracy within the class of all methods of a given order $p$, but we can use the error constant of the method which is defined by

$$
C=C_{p+1} / \sum_{i=0}^{k} \beta_{i}
$$

However, for both classes of formulas which we are considering the denominator of (4) is one, hence the error constant is the principal truncation error coefficient of the method.

The principal truncation error coefficients for the Adams formulas are available in [6] and those for the methods based on natural splines can be computed using (3) and the coefficients as given in [1]. Table 1 displays this data, which supports the observations made in [1] and [4].

TABLE 1. Principal truncation error coefficients

$\begin{array}{ccccc}\text { Order } & \text { Adams Predictor } & \text { Spline Predictor } & \text { Adams Corrector } & \text { Spline Corrector } \\ 2 & .41666667 & .29166667 & -.04166667 & -.02083333 \\ 3 & .375 & .21212121 & -.02638889 & -.01262626 \\ 4 & .34861111 & .16580206 & -.01875 & -.00781480 \\ 5 & .32986111 & .13534477 & -.01426918 & -.00518820 \\ 6 & .31559193 & .11386145 & -.01136739 & -.00365592\end{array}$

Computations were done in double precision on a CDC 6400 and then rounded to eight decimal places.

3. Finite-Difference Representations. The second technique we shall use to verify analytically the observations made in [1] and [4] requires us to write each of the multistep formulas in terms of forward differences. We proceed by observing that each formula under consideration represents an approximation of the difference

$$
y\left(x_{i+1}\right)-y\left(x_{i}\right)=h\left(f_{i}+\frac{1}{2 !} h f_{i}^{\prime}+\frac{1}{3 !} h^{2} f_{i}^{\prime \prime}+\cdots\right),
$$

which is obtained by replacing $h^{k} f_{i}^{(k)}$ by a finite-difference approximation.

The Adams formulas can be produced in this fashion by an orderly process which can be found in [2, pp. 7-11]. Unfortunately, no such process is available for the formulas based on natural splines, but we can achieve a similar representation by algebraic rearrangements. In the following tables, we present several formulas of each type written in terms of forward differences where $p$ denotes the order of the method. We shall use the standard notation for forward differences, namely

$$
\begin{aligned}
\Delta f_{i} & \equiv f_{i+1}-f_{i}, \\
\Delta^{k} f_{i} & \equiv \Delta^{k-1} f_{i+1}-\Delta^{k-1} f_{i} .
\end{aligned}
$$


TABLE 2. Forward-difference representations of Adams predictors

$$
\begin{array}{rr}
p=2 \quad y_{i+1}-y_{i}=h\left[f_{i}+\frac{1}{2 !} \Delta f_{i-1}\right] \\
p=3 \quad y_{i+1}-y_{i}=h\left[f_{i}+\frac{1}{2 !}\left(\Delta f_{i-2}+\frac{3}{2} \Delta^{2} f_{i-2}\right)+\frac{1}{3 !} \Delta^{2} f_{i-2}\right] \\
p=4 \quad y_{i+1}-y_{i}=h\left[f_{i}+\frac{1}{2 !}\left(\Delta f_{i-3}+\frac{5}{2} \Delta^{2} f_{i-3}+\frac{11}{6} \Delta^{3} f_{i-3}\right)\right. \\
\left.+\frac{1}{3 !}\left(\Delta^{2} f_{i-3}+2 \Delta^{3} f_{i-3}\right)+\frac{1}{4 !} \Delta^{3} f_{i-3}\right] \\
p=5 \quad y_{i+1}-y_{i}=h\left[f_{i}+\frac{1}{2 !}\left(\Delta f_{i-4}+\frac{7}{2} \Delta^{2} f_{i-4}+\frac{13}{3} \Delta^{3} f_{i-4}+\frac{25}{12} \Delta^{4} f_{i-4}\right)\right. \\
+\frac{1}{3 !}\left(\Delta^{2} f_{i-4}+3 \Delta^{3} f_{i-4}+\frac{35}{12} \Delta^{4} f_{i-4}\right) \\
\left.+\frac{1}{4 !}\left(\Delta^{3} f_{i-4}+\frac{5}{2} \Delta^{4} f_{i-4}\right)+\frac{1}{5 !} \Delta^{4} f_{i-4}\right]
\end{array}
$$

TABLE 3. Forward-difference representations of natural spline predictors

$$
\begin{aligned}
& p=2 \quad y_{i+1}-y_{i}=h\left[f_{i}+\frac{1}{2 !}\left(\Delta f_{i-2}+\frac{5}{4} \Delta^{2} f_{i-2}\right)\right] \\
& p=3 \quad y_{i+1}-y_{i}=h\left[f_{i}+\frac{1}{2 !}\left(\Delta f_{i-3}+\frac{5}{2} \Delta^{2} f_{i-3}+\frac{223}{132} \Delta^{3} f_{i-3}\right)\right. \\
& \left.+\frac{1}{3 !}\left(\Delta^{2} f_{i-3}+\frac{31}{22} \Delta^{3} f_{i-3}\right)\right] \\
& p=4 \quad y_{i+1}-y_{i}=h\left[f_{i}+\frac{1}{2 !}\left(\Delta f_{i-4}+\frac{7}{2} \Delta^{2} f_{i-4}+\frac{13}{3} \Delta^{3} f_{i-4}+\frac{3605}{1812} \Delta^{4} f_{i-4}\right)\right. \\
& +\frac{1}{3 !}\left(\Delta^{2} f_{i-4}+3 \Delta^{3} f_{i-4}+\frac{3011}{1208} \Delta^{4} f_{i-4}\right) \\
& \left.+\frac{1}{4 !}\left(\Delta^{3} f_{i-4}+\frac{233}{151} \Delta^{4} f_{i-4}\right)\right]
\end{aligned}
$$

TABLE 4. Forward-difference representations of Adams correctors

$$
\begin{aligned}
p=2 \quad y_{i}-y_{i-1} & =h\left[f_{i-1}+\frac{1}{2 !} \Delta f_{i-1}\right] \\
p=3 \quad y_{i}-y_{i-1} & =h\left[f_{i-1}+\frac{1}{2 !}\left(\Delta f_{i-2}+\frac{1}{2} \Delta^{2} f_{i-2}\right)+\frac{1}{3 !} \Delta^{2} f_{i-2}\right] \\
p=4 \quad y_{i}-y_{i-1} & =h\left[f_{i-1}+\frac{1}{2 !}\left(\Delta f_{i-3}+\frac{3}{2} \Delta^{2} f_{i-3}+\frac{1}{3} \Delta^{3} f_{i-3}\right)\right. \\
& \left.+\frac{1}{3 !}\left(\Delta^{2} f_{i-3}+\Delta^{3} f_{i-3}\right)+\frac{1}{4 !} \Delta^{3} f_{i-3}\right]
\end{aligned}
$$


(Table 4 continued)

$$
\begin{aligned}
p=5 \quad y_{i}-y_{i-1}=h\left[f_{i-1}\right. & +\frac{1}{2 !}\left(\Delta f_{i-4}+\frac{5}{2} \Delta^{2} f_{i-4}+\frac{11}{6} \Delta^{3} f_{i-4}+\frac{1}{4} \Delta^{4} f_{i-4}\right) \\
& +\frac{1}{3 !}\left(\Delta^{2} f_{i-4}+2 \Delta^{3} f_{i-4}+\frac{11}{12} \Delta^{4} f_{i-4}\right) \\
& \left.+\frac{1}{4 !}\left(\Delta^{3} f_{i-4}+\frac{3}{2} \Delta^{4} f_{i-4}\right)+\frac{1}{5 !} \Delta^{4} f_{i-4}\right]
\end{aligned}
$$

TABLE 5. Forward-difference representations of natural spline correctors

$$
\begin{aligned}
& p=2 \quad y_{i}-y_{i-1}=h\left[f_{i-1}+\frac{1}{2 !}\left(\Delta f_{i-2}+\frac{1}{2} \Delta^{2} f_{i-2}\right)\right. \\
& \left.+\frac{1}{3 !}\left(\frac{3}{2} \Delta^{2} f_{i-2}\right)+\frac{1}{4 !}\left(-\frac{3}{2} \Delta^{2} f_{i-2}\right)\right] \\
& p=3 \quad y_{i}-y_{i-1}=h\left[f_{i-1}+\frac{1}{2 !}\left(\Delta f_{i-3}+\frac{3}{2} \Delta^{2} f_{i-3}+\frac{141}{396} \Delta^{3} f_{i-3}\right)\right. \\
& +\frac{1}{3 !}\left(\Delta^{2} f_{i-3}+\frac{73}{66} \Delta^{3} f_{i-3}\right)+\frac{1}{4 !}\left(\frac{10}{11} \Delta^{3} f_{i-3}\right) \\
& \left.+\frac{1}{5 !}\left(-\frac{20}{11} \Delta^{3} f_{i-3}\right)+\frac{1}{6 !}\left(\frac{20}{11} \Delta^{3} f_{i-3}\right)\right] \\
& p=4 \quad y_{i}-y_{i-1}=h\left[f_{i-1}+\frac{1}{2 !}\left(\Delta f_{i-4}+\frac{5}{2} \Delta^{2} f_{i-4}+\frac{11}{6} \Delta^{3} f_{i-4}+\frac{1925}{7248} \Delta^{4} f_{i-4}\right)\right. \\
& +\frac{1}{3 !}\left(\Delta^{2} f_{i-4}+2 \Delta^{3} f_{i-4}+\frac{1168}{1208} \Delta^{4} f_{i-4}\right) \\
& +\frac{1}{4 !}\left(\Delta^{3} f_{i-4}+\frac{7025}{4832} \Delta^{4} f_{i-4}\right)+\frac{1}{5 !}\left(\frac{210}{604} \Delta^{4} f_{i-4}\right) \\
& \left.+\frac{1}{6 !}\left(-\frac{315}{302} \Delta^{4} f_{i-4}\right)+\frac{1}{7 !}\left(\frac{315}{151} \Delta^{4} f_{i-4}\right)+\frac{1}{8 !}\left(-\frac{315}{151} \Delta^{4} f_{i-4}\right)\right]
\end{aligned}
$$

We can now observe that in Tables 3 and 5 the approximations to the derivatives $f_{i}^{(k)}$ in a $p$ th order formula are, except for the last terms, the same as those for a $(p+1)$ st order formula in Tables 2 and 4, respectively. This implies that the $p$ th order natural spline formulas do much better than the corresponding Adams formulas because of better derivative approximations.

4. Conclusions. The results presented in the previous two sections together with the comparisons of the $L-2$ norms of the Peano kernels given in [1] provide a rigorous foundation for the statement that, for the cases considered $(p=2,3,4)$, a $p$ th order natural spline linear multistep formula will yield more accurate approximations to the solution of (1) than a $p$ th order Adams formula, but not quite as good as the $(p+1)$ st order Adams methods, for sufficiently smooth functions. These results suggest a similar comparison for the general case. 
Department of Mathematics

Temple University

Philadelphia, Pennsylvania 19122

1. G. D. ANDRIA, G. D. BYRNE \& D. R. HILL, "Integration formulas and schemes based on $g$-splines," Math. Comp., v. 27, 1973, pp. 831-838; with microfiche supplement in Addendum Section.

2. L. FOX, The Numerical Solution of Two-Point Boundary Problems in Ordinary Differential Equations, Oxford Univ. Press, New York, 1957. MR 21 \#972.

3. P. HENRICI, Discrete Variable Methods in Ordinary Differential Equations, Wiley, New York, 1962. MR 24 \#B1772.

4. D. R. HILL, An Approach to the Numerical Solution of Delay Differential Equations, Ph. D. Thesis, University of Pittsburgh, 1973.

5. T. E. HULL \& A. C. R. NEWBERY, "Corrector formulas for multi-step integration methods," J. Soc. Indust. Appl. Math., v. 10, 1962, pp. 351-369. MR 27 \#2130.

6. L. LAPIDUS \& J. H. SEINFELD, Numerical Solution of Ordinary Differential Equations, Math. in Sci. and Engineering, vol. 74, Academic Press, New York, 1971. MR 43 \#7073. 